\title{
On $r$-circulant matrices with Horadam numbers having arithmetic indices
}

\author{
Aldous Cesar F. Bueno \\ Mathematics Unit, Philippine Science High School - Central Luzon Campus \\ Lily Hill, Clark Freeport Zone, Pampanga, Philippines \\ e-mails: afbueno@clc.pshs.edu.ph, aldzczrcirc@gmail.com
}

Received: 2 January 2020

Revised: 25 April 2020

Accepted: 27 April 2020

\begin{abstract}
We investigate an $r$-circulant matrix whose entries are Horadam numbers having arithmetic indices. We then solve for the eigenvalues, determinant, Euclidean norm and spectral norm of the matrix. Lastly, we present some special cases and some results on identities and divisibility.
\end{abstract}

Keywords: Horadam Numbers, $r$-circulant matrix, Eigenvalue, Determinant, Euclidean norm, Spectral norm.

2010 Mathematics Subject Classification: 11B05, 15B36, $11 B 39$.

\section{Introduction}

Given a nonzero complex number $r$ and a finite sequence $\left\{s_{k}\right\}_{k=0}^{n-1}$, a certain $r$-circulant matrix can be formed. From this type of matrix, it has been a custom to determine and investigate its eigenvalues, determinant, inverse, Euclidean norm and spectral norm.

The cases where $r= \pm 1$ partnered with a sequence satisfying a recurrence relation are the ones that are usually explored. Some studies that involve such approach are that of Bahsi and Solak [1], Bozkurt [3,5], Bozkurt and Tam [4], Bueno [6-13], Civciv and Turkmen [16], Lind [19], Majumdar [20], Nalli and Sen [21], Shen and Cen [23] and Yalciner [25].

There are also studies that revolve on $r$-circulant matrices having special sequences. In Radicic [22], $r$-circulant matrices involving geometric sequences were considered. Bozkurt and Tam [5] worked on the determinants and inverses of $r$-circulant matrices with Horadam numbers. The bounds of the spectral norms of $r$-circulant matrices with $k$-Fibonacci and $k$-Lucas 
numbers were derived by Shen and Cen [24]. In Bueno [14], $r$-circulant matrices with Fibonacci and Lucas numbers having arithmetic indices were introduced and some properties of the eigenvalues, determinants and norms of the matrices were established.

In this research, we will examine the $r$-circulant matrix given by

$$
M=\left(\begin{array}{cccccc}
W_{s} & W_{s+t} & W_{s+2 t} & \ldots & W_{s+(n-2) t} & W_{s+(n-1) t} \\
r W_{s+(n-1) t} & W_{s} & W_{s+t} & \ldots & W_{s+(n-3) t} & W_{s+(n-2) t} \\
r W_{s+(n-2) t} & r W_{s+(n-1) t} & W_{s} & \ldots & W_{s+(n-4) t} & W_{s+(n-3) t} \\
\vdots & \vdots & \vdots & \ddots & \vdots & \vdots \\
r W_{s+2 t} & r W_{s+3 t} & r W_{s+4 t} & \ldots & W_{s} & W_{s+t} \\
r W_{s+t} & r W_{s+2 t} & r W_{s+3 t} & \ldots & r W_{s+(n-1) t} & W_{s}
\end{array}\right) .
$$

In the matrix above, $W_{j}$ s are Horadam numbers and $s, t \in \mathbb{Z}$. Observe that the indices of the Horadam numbers in the matrix form an arithmetic sequence and not only limited to nonnegative values. Our objective is to derive for the formulas of the eigenvalues, determinant, Euclidean norm and spectral norm of the matrix. We will also consider some special cases of the Horadam numbers and establish some identities and results on divisibility.

\section{Preliminaries}

\subsection{The Horadam sequence}

We first define the Horadam sequence and enumerate some of its special cases.

Definition 2.1 ([18]). The sequence given by $\left\{W_{k}\right\}_{k=0}^{+\infty}$ that satisfy the following recurrence relation

$$
W_{n}(a, b, p, q)=W_{n}=\left\{\begin{array}{ll}
a & \text { if } n=0 \\
b & \text { if } n=1 \\
p W_{n-1}-q W_{n-2} & \text { if } n \geq 2
\end{array},\right.
$$

where $a, b, p, q \in \mathbb{R}$, is called the Horadam sequence.

For the $n$-th Horadam number, we have the Binet's formula given by

$$
W_{n}=A \alpha^{n}+B \beta^{n},
$$

where $\alpha=\frac{p+\sqrt{p^{2}-4 q}}{2}, \beta=\frac{p-\sqrt{p^{2}-4 q}}{2}, A=\frac{b-a \beta}{\alpha-\beta}$ and $B=\frac{a \alpha-b}{\alpha-\beta}$.

The following well-known sequences are all special cases of the Horadam sequence.

1. Fibonacci sequence: $\left\{F_{k}\right\}_{k=0}^{+\infty}$

- Recurrence relation:

$$
F_{n}=W_{n}(0,1,1,-1)= \begin{cases}0 & \text { if } n=0 \\ 1 & \text { if } n=1 \\ F_{n-1}+F_{n-2} & \text { if } n \geq 2\end{cases}
$$


- Binet's formula:

$$
F_{n}=\frac{1}{\sqrt{5}}\left[\left(\frac{1+\sqrt{5}}{2}\right)^{n}-\left(\frac{1-\sqrt{5}}{2}\right)^{n}\right]
$$

2. Lucas sequence: $\left\{L_{k}\right\}_{k=0}^{+\infty}$

- Recurrence relation

$$
L_{n}=W_{n}(2,1,1,-1)= \begin{cases}2 & \text { if } n=0 \\ 1 & \text { if } n=1 \\ L_{n-1}+L_{n-2} & \text { if } n \geq 2\end{cases}
$$

- Binet's formula:

$$
L_{n}=\left(\frac{1+\sqrt{5}}{2}\right)^{n}+\left(\frac{1-\sqrt{5}}{2}\right)^{n}
$$

3. Jacobsthal sequence: $\left\{J_{k}\right\}_{k=0}^{+\infty}$

- Recurrence relation:

$$
J_{n}=W_{n}(0,1,1,-2)= \begin{cases}0 & \text { if } n=0 \\ 1 & \text { if } n=1 \\ J_{n-1}+2 J_{n-2} & \text { if } n \geq 2\end{cases}
$$

- Binet's formula:

$$
J_{n}=\frac{1}{3}\left[2^{n}-(-1)^{n}\right]
$$

4. Jacobsthal-Lucas sequence: $\left\{K_{k}\right\}_{k=0}^{+\infty}$

- Recurrence relation:

$$
K_{n}=W_{n}(2,1,1,-2)= \begin{cases}2 & \text { if } n=0 \\ 1 & \text { if } n=1 \\ K_{n-1}+2 K_{n-2} & \text { if } n \geq 2\end{cases}
$$

- Binet's formula:

$$
K_{n}=2^{n}+(-1)^{n}
$$

5. Pell sequence: $\left\{P_{k}\right\}_{k=0}^{+\infty}$

- Recurrence relation:

$$
P_{n}=W_{n}(0,1,2,-1)= \begin{cases}0 & \text { if } n=0 \\ 1 & \text { if } n=1 \\ 2 P_{n-1}+P_{n-2} & \text { if } n \geq 2\end{cases}
$$

- Binet's formula:

$$
P_{n}=\frac{1}{2 \sqrt{2}}\left[(1+\sqrt{2})^{n}-(1-\sqrt{2})^{n}\right]
$$


6. Pell-Lucas sequence: $\left\{Q_{k}\right\}_{k=0}^{+\infty}$

- Recurrence relation:

$$
Q_{n}=W_{n}(2,2,2,-1)= \begin{cases}2 & \text { if } n=0 \\ 2 & \text { if } n=1 \\ 2 Q_{n-1}+Q_{n-2} & \text { if } n \geq 2\end{cases}
$$

- Binet's formula:

$$
Q_{n}=(1+\sqrt{2})^{n}+(1-\sqrt{2})^{n}
$$

\subsection{Notations}

After the discussion of Horadam numbers, we now introduce some notations. For the rest of the paper we will use the following notations:

- $M(r, \vec{W})=M$, the $r$-circulant matrix with Horadam numbers having arithmetic indices

- $\Delta[r, \vec{W}]:=$ determinant of $M(r, \vec{W})$

- $\lambda_{m}[r, \vec{W}]:=$ an eigenvalue of $M(r, \vec{W}), m=0,1, \ldots, n-1$

- $\|M(r, \vec{W})\|_{E}:=$ Euclidean norm of $M(r, \vec{W})$

- $\|M(r, \vec{W})\|_{2}:=$ spectral norm of $M(r, \vec{W})$

- $W_{-n}=A \alpha^{-n}+B \beta^{-n}$

- $V_{n}=\alpha^{n}+\beta^{n}$

- $U_{n}=B \alpha^{n}+A \beta^{n}$

- $\rho=|r|^{1 / n}\left[\cos \left(\frac{\theta+2 \pi j}{n}\right)+i \sin \left(\frac{\theta+2 \pi j}{n}\right)\right]$, an $n$-th root of $r, j=0,1, \ldots, n-1$

- $\omega=e^{2 \pi i / n}=\cos \left(\frac{2 \pi j}{n}\right)+i \sin \left(\frac{2 \pi j}{n}\right)$, an $n$-th root of unity, $j=0,1, \ldots, n-1$

\subsection{The $r$-circulant matrices}

We now define the $r$-circulant and provide some of its properties.

Definition 2.2. An r-circulant matrix is a matrix of the form

$$
M(r, \vec{c})=\left(\begin{array}{cccccc}
c_{0} & c_{1} & c_{2} & \ldots & c_{n-2} & c_{n-1} \\
r c_{n-1} & c_{0} & c_{1} & \ldots & c_{n-3} & c_{n-2} \\
r c_{n-2} & r c_{n-1} & c_{0} & \ldots & c_{n-4} & c_{n-3} \\
\vdots & \vdots & \vdots & \ddots & \vdots & \vdots \\
r c_{2} & r c_{3} & r c_{4} & \ldots & c_{0} & c_{1} \\
r c_{1} & r c_{2} & r c_{3} & \ldots & r c_{n-1} & c_{0}
\end{array}\right)
$$

where $r \in \mathbb{C} \backslash\{0\}$ and $\vec{c}=\left(c_{0}, c_{1}, c_{2}, \ldots, c_{n-2}, c_{n-1}\right) \in \mathbb{R}^{n}$. The vector $\vec{c}$ is called the circulant vector and it determines the r-circulant matrix. 
The 1-circulant matrices and -1-circulant matrices are called the right circulant matrices and skew right circulant matrices, respectively. In general, $r$-circulant matrices are Toeplitz matrices [17]; they are diagonal-constant matrices.

Here are some properties of the $r$-circulant matrices that we will use.

We start with the eigenvalues.

Theorem 2.3 ( [15]). The eigenvalues of $M(r, \vec{c})$ are given by

$$
\lambda_{m}[r, \vec{c}]=\sum_{k=0}^{n-1} c_{k}\left(\rho \omega^{-m}\right)^{k},
$$

where $m=0,1, \ldots, n-1, \rho$ is an $n$-th root of $r$ and $\omega$ is an $n$-th root of unity.

Basically, the right-hand side of (5) is the Generalized Discrete Fourier Transform (GDFT) of $\vec{c}$. For $r=1$ we have the following result from Gray [17].

Theorem 2.4 ([17]). The eigenvalues of the right circulant matrix $M(1, \vec{c})$ are given by

$$
\lambda_{m}[1, \vec{c}]=\sum_{k=0}^{n-1} c_{k} \omega^{-m k}
$$

where $m=0,1, \ldots, n-1$.

Note that, this is just the Discrete Fourier Transform (DFT) of $\vec{c}$. Since right circulant matrices are related to the DFT via their eigenvalues, they appear in signal processing theory and coding theory.

From the eigenvalues, it follows that the determinant is given by

$$
\Delta[r, \vec{c}]=\prod_{m=0}^{n-1}\left[\sum_{k=0}^{n-1} c_{k}\left(\rho \omega^{-m}\right)^{k}\right] .
$$

For the spectral norm, we have

$$
\|M(r, \vec{c})\|_{2}=\max _{0 \leq m \leq n-1}\left\{\left|\sum_{k=0}^{n-1} c_{k}\left(\rho \omega^{-m}\right)^{k}\right|\right\} .
$$

Finally, for the Euclidean norm, we have the following result.

Lemma 2.5 ([14]). The Euclidean norm of $M(r, \vec{c})$ is given by

$$
\|M(r, \vec{W})\|_{E}=\sqrt{\sum_{k=0}^{n-1}\left|c_{k}\right|^{2}\left[n-k\left(1-|r|^{2}\right)\right]}
$$




\section{Main results}

\subsection{The eigenvalues of $M(r, \vec{W})$}

For the eigenvalues $M(r, \vec{W})$ we have the following results.

Theorem 3.1. The eigenvalues of $M[r, \vec{W}]$ are given by

$$
\lambda_{m}[r, \vec{W}]= \begin{cases}\frac{W_{s}-r W_{s+n t}-\left(q^{s} U_{t-s}-r q^{t} W_{s+(n-1) t}\right) \rho \omega^{-m}}{\left(1-\alpha^{t} \rho \omega^{-m}\right)\left(1-\beta^{t} \rho \omega^{-m}\right)}, & s<t \\ \frac{W_{s}-r W_{(n+1) s}-q^{s}\left(a-r W_{n s}\right) \rho \omega^{-m}}{\left(1-\alpha^{s} \rho \omega^{-m}\right)\left(1-\beta^{s} \rho \omega^{-m}\right)}, & s=t \\ \frac{W_{s}-r W_{s+n t}-q^{t}\left(W_{s-t}-r W_{s+(n-1) t}\right) \rho \omega^{-m}}{\left(1-\alpha^{t} \rho \omega^{-m}\right)\left(1-\beta^{t} \rho \omega^{-m}\right)}, & s>t\end{cases}
$$

where $m=0,1, \ldots, n-1$.

Proof.

$$
\begin{aligned}
\lambda_{m}[r, \vec{W}] & =\sum_{k=0}^{n-1} W_{s+k t}\left(\rho \omega^{-m}\right)^{k} \\
& =\sum_{k=0}^{n-1}\left[A \alpha^{s+k t}+B \beta^{s+k t}\right]\left(\rho \omega^{-m}\right)^{k} \\
& =A \alpha^{s} \sum_{k=0}^{n-1}\left(\alpha^{t} \rho \omega^{-m}\right)^{k}+B \beta^{s} \sum_{k=0}^{n-1}\left(\beta^{t} \rho \omega^{-m}\right)^{k} \\
& =A \alpha^{s}\left(\frac{1-r \alpha^{n t}}{1-\alpha^{t} \rho \omega^{-m}}\right)+B \beta^{s}\left(\frac{1-r \beta^{n t}}{1-\beta^{t} \rho \omega^{-m}}\right) \\
& =\frac{\left(A \alpha^{s}-r A \alpha^{s+n t}\right)\left(1-\beta^{t} \rho \omega^{-m}\right)+\left(B \beta^{s}-r B \beta^{s+n t}\right)\left(1-\alpha^{t} \rho \omega^{-m}\right)}{\left(1-\alpha^{t} \rho \omega^{-m}\right)\left(1-\beta^{t} \rho \omega^{-m}\right)} \\
& =\frac{\left[A \alpha^{s}+B \beta^{s}\right]-r\left[A \alpha^{s+n t}+B \beta^{s+n t}\right]}{\left(1-\alpha^{t} \rho \omega^{-m}\right)\left(1-\beta^{t} \rho \omega^{-m}\right)}- \\
& \frac{\left[\left(A \alpha^{s} \beta^{t}+B \alpha^{t} \beta^{s}\right)+r q^{t}\left(A \alpha^{s+(n-1) t}+B \beta^{s+(n-1) t}\right)\right] \rho \omega^{-m}}{\left(1-\alpha^{t} \rho \omega^{-m}\right)\left(1-\beta^{t} \rho \omega^{-m}\right)} \\
= & \frac{W_{s}-r W_{s+n t}-\left[\left(A \alpha^{s} \beta^{t}+B \alpha^{t} \beta^{s}\right)-r q^{t} W_{s+(n-1) t}\right] \rho \omega^{-m}}{\left(1-\alpha^{t} \rho \omega^{-m}\right)\left(1-\beta^{t} \rho \omega^{-m}\right)}
\end{aligned}
$$

Note that

$$
A \alpha^{s} \beta^{t}+B \alpha^{t} \beta^{s}= \begin{cases}q^{s} U_{t-s}, & s<t \\ a q^{s}, & s=t \\ q^{t} W_{s-t}, & s>t\end{cases}
$$


Using the equation above, we obtain

$$
\lambda_{m}[r, \vec{W}]= \begin{cases}\frac{W_{s}-r W_{s+n t}-\left[q^{s} U_{t-s}-r q^{t} W_{s+(n-1) t}\right] \rho \omega^{-m}}{\left(1-\alpha^{t} \rho \omega^{-m}\right)\left(1-\beta^{t} \rho \omega^{-m}\right)}, & s<t \\ \frac{W_{s}-r W_{(n+1) s}-q^{s}\left[a-r W_{n s}\right] \rho \omega^{-m}}{\left(1-\alpha^{s} \rho \omega^{-m}\right)\left(1-\beta^{s} \rho \omega^{-m}\right)}, & s=t . \\ \frac{W_{s}-r W_{s+n t}-q^{t}\left[W_{s-t}-r W_{s+(n-1) t}\right] \rho \omega^{-m}}{\left(1-\alpha^{t} \rho \omega^{-m}\right)\left(1-\beta^{t} \rho \omega^{-m}\right)}, & s>t\end{cases}
$$

Here are some special cases of Theorem 3.1.

\section{Corollary 3.2.}

$$
\lambda_{m}[r, \vec{F}]= \begin{cases}\frac{F_{s}-r F_{s+n t}-\left[(-1)^{s} F_{t-s}-(-1)^{t} r F_{s+(n-1) t}\right] \rho \omega^{-m}}{\left(1-\alpha^{t} \rho \omega^{-m}\right)\left(1-\beta^{t} \rho \omega^{-m}\right)}, & s<t \\ \frac{F_{s}-r F_{(n+1) s}+(-1)^{s} r F_{n s} \rho \omega^{-m}}{\left(1-\alpha^{s} \rho \omega^{-m}\right)\left(1-\beta^{s} \rho \omega^{-m}\right)}, & s=t \\ \frac{F_{s}-r F_{s+n t}-(-1)^{t}\left[F_{s-t}-r F_{s+(n-1) t}\right] \rho \omega^{-m}}{\left(1-\alpha^{t} \rho \omega^{-m}\right)\left(1-\beta^{t} \rho \omega^{-m}\right)}, & s>t\end{cases}
$$

where $\alpha=\frac{1+\sqrt{5}}{2}$ and $\beta=\frac{1-\sqrt{5}}{2}$.

\section{Corollary 3.3.}

$$
\lambda_{m}[r, \vec{L}]= \begin{cases}\frac{L_{s}-r L_{s+n t}-\left[(-1)^{s} L_{t-s}-(-1)^{t} r L_{s+(n-1) t}\right] \rho \omega^{-m}}{\left(1-\alpha^{t} \rho \omega^{-m}\right)\left(1-\beta^{t} \rho \omega^{-m}\right)}, & s<t \\ \frac{L_{s}-r L_{(n+1) s}-(-1)^{s}\left[2-r L_{n s}\right] \rho \omega^{-m}}{\left(1-\alpha^{s} \rho \omega^{-m}\right)\left(1-\beta^{s} \rho \omega^{-m}\right)}, & s=t \\ \frac{L_{s}-r L_{s+n t}-(-1)^{t}\left[L_{s-t}-r L_{s+(n-1) t}\right] \rho \omega^{-m}}{\left(1-\alpha^{t} \rho \omega^{-m}\right)\left(1-\beta^{t} \rho \omega^{-m}\right)}, & s>t\end{cases}
$$

where $\alpha=\frac{1+\sqrt{5}}{2}$ and $\beta=\frac{1-\sqrt{5}}{2}$.

\section{Corollary 3.4.}

$$
\lambda_{m}[r, \vec{J}]= \begin{cases}\frac{J_{s}-r J_{s+n t}+\left[(-2)^{s} J_{t-s}+(-2)^{t} r J_{s+(n-1) t}\right] \rho \omega^{-m}}{\left(1-2^{t} \rho \omega^{-m}\right)\left(1-(-1)^{t} \rho \omega^{-m}\right)}, & s<t \\ \frac{J_{s}-r J_{(n+1) s}+(-2)^{s} r J_{n s} \rho \omega^{-m}}{\left(1-2^{s} \rho \omega^{-m}\right)\left(1-(-1)^{s} \rho \omega^{-m}\right)}, & s=t \\ \frac{J_{s}-r J_{s+n t}-(-2)^{t}\left[J_{s-t}-r J_{s+(n-1) t}\right] \rho \omega^{-m}}{\left(1-2^{t} \rho \omega^{-m}\right)\left(1-(-1)^{t} \rho \omega^{-m}\right)}, & s>t\end{cases}
$$




\section{Corollary 3.5.}

$$
\lambda_{m}[r, \vec{K}]= \begin{cases}\frac{K_{s}-r K_{s+n t}-\left[(-2)^{s} K_{t-s}-(-2)^{t} r K_{s+(n-1) t}\right] \rho \omega^{-m}}{\left(1-2^{t} \rho \omega^{-m}\right)\left(1-(-1)^{t} \rho \omega^{-m}\right)}, & s<t \\ \frac{K_{s}-r K_{(n+1) s}-(-2)^{s}\left[2-r K_{n s}\right] \rho \omega^{-m}}{\left(1-2^{s} \rho \omega^{-m}\right)\left(1-(-1)^{s} \rho \omega^{-m}\right)}, & s=t \\ \frac{K_{s}-r K_{s+n t}-(-2)^{t}\left[K_{s-t}-r K_{s+(n-1) t}\right] \rho \omega^{-m}}{\left(1-2^{t} \rho \omega^{-m}\right)\left(1-(-1)^{t} \rho \omega^{-m}\right)}, & s>t\end{cases}
$$

\section{Corollary 3.6.}

$$
\lambda_{m}[r, \vec{P}]= \begin{cases}\frac{P_{s}-r P_{s+n t}-\left[(-1)^{s+1} P_{t-s}-(-1)^{t} r P_{s+(n-1) t}\right] \rho \omega^{-m}}{\left(1-\alpha^{t} \rho \omega^{-m}\right)\left(1-\beta^{t} \rho \omega^{-m}\right)}, & s<t \\ \frac{P_{s}-r P_{(n+1) s}+(-1)^{s} r P_{n s} \rho \omega^{-m}}{\left(1-\alpha^{s} \rho \omega^{-m}\right)\left(1-\beta^{s} \rho \omega^{-m}\right)}, & s=t \\ \frac{P_{s}-r P_{s+n t}-(-1)^{t}\left[P_{s-t}-r P_{s+(n-1) t}\right] \rho \omega^{-m}}{\left(1-\alpha^{t} \rho \omega^{-m}\right)\left(1-\beta^{t} \rho \omega^{-m}\right)}, & s>t\end{cases}
$$

where $\alpha=1+\sqrt{2}$ and $\beta=1-\sqrt{2}$.

\section{Corollary 3.7.}

$$
\lambda_{m}[r, \vec{Q}]= \begin{cases}\frac{Q_{s}-r Q_{s+n t}-\left[(-1)^{s} Q_{t-s}-(-1)^{t} r Q_{s+(n-1) t}\right] \rho \omega^{-m}}{\left(1-\alpha^{t} \rho \omega^{-m}\right)\left(1-\beta^{t} \rho \omega^{-m}\right)}, & s<t \\ \frac{Q_{s}-r Q_{(n+1) s}-(-1)^{s}\left[2-r Q_{n s}\right] \rho \omega^{-m}}{\left(1-\alpha^{s} \rho \omega^{-m}\right)\left(1-\beta^{s} \rho \omega^{-m}\right)}, & s=t \\ \frac{Q_{s}-r Q_{s+n t}-(-1)^{t}\left[Q_{s-t}-r Q_{s+(n-1) t}\right] \rho \omega^{-m}}{\left(1-\alpha^{t} \rho \omega^{-m}\right)\left(1-\beta^{t} \rho \omega^{-m}\right)}, & s>t\end{cases}
$$

where $\alpha=1+\sqrt{2}$ and $\beta=1-\sqrt{2}$.

\subsection{The determinant of $M(r, \vec{W})$}

We now discuss the results on the determinant of $M(r, \vec{W})$.

\section{Theorem 3.8.}

$$
\Delta[r, \vec{W}]= \begin{cases}\frac{\left(W_{s}-r W_{s+n t}\right)^{n}-r\left[q^{s} U_{t-s}-r q^{t} W_{s+(n-1) t}\right]^{n}}{1-r V_{n t}+r^{2} q^{n t}}, & s<t \\ \frac{\left(W_{s}-r W_{(n+1) s}\right)^{n}-r q^{n s}\left[a-r W_{n s}\right]^{n}}{1-r V_{n s}+r^{2} q^{n s}}, & s=t \\ \frac{\left(W_{s}-r W_{s+n t}\right)^{n}-r q^{n t}\left[W_{s-t}-r W_{s+(n-1) t}\right]^{n}}{1-r V_{n t}+r^{2} q^{n t}}, & s>t\end{cases}
$$


Proof.

$$
\begin{aligned}
& \Delta[r, \vec{W}]=\prod_{m=0}^{n-1} \lambda_{m}[r, \vec{W}] \\
& = \begin{cases}\prod_{m=0}^{n-1} \frac{W_{s}-r W_{s+n t}-\left[q^{s} U_{t-s}-r q^{t} W_{s+(n-1) t}\right] \rho \omega^{-m}}{\left(1-\alpha^{t} \rho \omega^{-m}\right)\left(1-\beta^{t} \rho \omega^{-m}\right)}, & s<t \\
\prod_{m=0}^{n-1} \frac{W_{s}-r W_{(n+1) s}-q^{s}\left[a-r W_{n s}\right] \rho \omega^{-m}}{\left(1-\alpha^{s} \rho \omega^{-m}\right)\left(1-\beta^{s} \rho \omega^{-m}\right)}, & s=t \\
\prod_{m=0}^{n-1} \frac{W_{s}-r W_{s+n t}-q^{t}\left[W_{s-t}-r W_{s+(n-1) t}\right] \rho \omega^{-m}}{\left(1-\alpha^{t} \rho \omega^{-m}\right)\left(1-\beta^{t} \rho \omega^{-m}\right)}, & s>t\end{cases} \\
& = \begin{cases}\frac{\left(W_{s}-r W_{s+n t}\right)^{n}-r\left[q^{s} U_{t-s}-r q^{t} W_{s+(n-1) t}\right]^{n}}{\left(1-r \alpha^{n t}\right)\left(1-r \beta^{n t}\right)}, & s<t \\
\frac{\left(W_{s}-r W_{(n+1) s}\right)^{n}-r q^{n s}\left[a-r W_{n s}\right]^{n}}{\left(1-r \alpha^{n s}\right)\left(1-r \beta^{n s}\right)}, & s=t \\
\frac{\left(W_{s}-r W_{s+n t}\right)^{n}-r q^{t}\left[W_{s-t}-r W_{s+(n-1) t}\right]^{n}}{\left(1-r \alpha^{n t}\right)\left(1-r \beta^{n t}\right)}, & s>t\end{cases} \\
& = \begin{cases}\frac{\left(W_{s}-r W_{s+n t}\right)^{n}-r\left[q^{s} U_{t-s}-r q^{t} W_{s+(n-1) t}\right]^{n}}{1-r V_{n t}+r^{2} q^{n t}}, & s<t \\
\frac{\left(W_{s}-r W_{(n+1) s}\right)^{n}-r q^{n s}\left[a-r W_{n s}\right]^{n}}{1-r V_{n s}+r^{2} q^{n s}}, & s=t \\
\frac{\left(W_{s}-r W_{s+n t}\right)^{n}-r q^{n t}\left[W_{s-t}-r W_{s+(n-1) t}\right]^{n}}{1-r V_{n t}+r^{2} q^{n t}}, & s>t\end{cases}
\end{aligned}
$$

Here are some special cases of Theorem 3.8.

\section{Corollary 3.9.}

$$
\Delta[r, \vec{F}]= \begin{cases}\frac{\left(F_{s}-r F_{s+n t}\right)^{n}-r\left[(-1)^{s} F_{t-s}-(-1)^{t} r F_{s+(n-1) t}\right]^{n}}{1-r L_{n t}+(-1)^{n t} r^{2}}, & s<t \\ \frac{\left(F_{s}-r F_{(n+1) s}\right)^{n}-(-1)^{n s} r\left(-r F_{n s}\right)^{n}}{1-r L_{n s}+(-1)^{n s} r^{2}}, & s=t \\ \frac{\left(F_{s}-r F_{s+n t}\right)^{n}-(-1)^{n t} r\left(F_{s-t}-r F_{s+(n-1) t}\right)^{n}}{1-r L_{n t}+(-1)^{n t} r^{2}}, & s>t\end{cases}
$$

\section{Corollary 3.10.}

$$
\Delta[r, \vec{L}]= \begin{cases}\frac{\left(L_{s}-r L_{s+n t}\right)^{n}-r\left[(-1)^{s} L_{t-s}-(-1)^{t} r L_{s+(n-1) t}\right]^{n}}{1-r L_{n t}+(-1)^{n t} r^{2}}, & s<t \\ \frac{\left(L_{s}-r L_{(n+1) s}\right)^{n}-(-1)^{n s} r\left[2-r L_{n s}\right]^{n}}{1-r L_{n s}+(-1)^{n s} r^{2}}, & s=t \\ \frac{\left(L_{s}-r L_{s+n t}\right)^{n}-(-1)^{n t} r\left[L_{s-t}-r L_{s+(n-1) t}\right]^{n}}{1-r L_{n t}+(-1)^{n t} r^{2}}, & s>t\end{cases}
$$




\section{Corollary 3.11.}

$$
\Delta[r, \vec{J}]= \begin{cases}\frac{\left(J_{s}-r J_{s+n t}\right)^{n}-r\left[(-2)^{s+1} J_{t-s}-(-2)^{t} r J_{s+(n-1) t}\right]^{n}}{1-r K_{n t}+(-2)^{n t} r^{2}}, & s<t \\ \frac{\left(J_{s}-r J_{(n+1) s}\right)^{n}-(-2)^{n s} r\left(-r J_{n s}\right)^{n}}{1-r K_{n s}+(-2)^{n s} r^{2}}, & s=t \\ \frac{\left(J_{s}-r J_{s+n t}\right)^{n}-(-2)^{n t} r\left[J_{s-t}-r J_{s+(n-1) t}\right]^{n}}{1-r K_{n t}+(-2)^{n t} r^{2}}, & s>t\end{cases}
$$

\section{Corollary 3.12.}

$$
\Delta[r, \vec{K}]= \begin{cases}\frac{\left(K_{s}-r K_{s+n t}\right)^{n}-r\left[(-2)^{s} K_{t-s}-(-2)^{t} r K_{s+(n-1) t}\right]^{n}}{1-r K_{n t}+(-2)^{n t} r^{2}}, & s<t \\ \frac{\left(K_{s}-r K_{(n+1) s}\right)^{n}-(-2)^{n s} r\left[2-r K_{n s}\right]^{n}}{1-r K_{n s}+(-2)^{n s} r^{2}}, & s=t \\ \frac{\left(K_{s}-r K_{s+n t}\right)^{n}-(-2)^{n t} r\left[K_{s-t}-r K_{s+(n-1) t}\right]^{n}}{1-r K_{n t}+(-2)^{n t} r^{2}}, & s>t\end{cases}
$$

\section{Corollary 3.13.}

$$
\Delta[r, \vec{P}]= \begin{cases}\frac{\left(P_{s}-r P_{s+n t}\right)^{n}-r\left[(-1)^{s+1} P_{t-s}-(-1)^{t} r P_{s+(n-1) t}\right]^{n}}{1-r Q_{n t}+(-1)^{n t} r^{2}}, & s<t \\ \frac{\left(P_{s}-r P_{(n+1) s}\right)^{n}-(-1)^{n s} r\left(-r P_{n s}\right)^{n}}{1-r Q_{n s}+(-1)^{n s} r^{2}}, & s=t \\ \frac{\left(P_{s}-r P_{s+n t}\right)^{n}-(-1)^{n t} r\left[P_{s-t}-r P_{s+(n-1) t}\right]^{n}}{1-r Q_{n t}+(-1)^{n t} r^{2}}, & s>t\end{cases}
$$

Corollary 3.14.

$$
\Delta[r, \vec{Q}]= \begin{cases}\frac{\left(Q_{s}-r Q_{s+n t}\right)^{n}-r\left[(-1)^{s} Q_{t-s}-(-1)^{t} r Q_{s+(n-1) t}\right]^{n}}{1-r Q_{n t}+(-1)^{n t} r^{2}}, & s<t \\ \frac{\left(Q_{s}-r Q_{(n+1) s}\right)^{n}-(-1)^{n s} r\left[2-r Q_{n s}\right]^{n}}{1-r Q_{n s}+(-1)^{n s} r^{2}}, & s=t \\ \frac{\left(Q_{s}-r Q_{s+n t}\right)^{n}-(-1)^{n t} r\left[Q_{s-t}-r Q_{s+(n-1) t}\right]^{n}}{1-r Q_{n t}+(-1)^{n t} r^{2}}, & s>t\end{cases}
$$

\subsection{Sum identities}

From the eigenvalues of $M(r, \vec{W})$, if we choose $m=0, r=1$ and $\rho=1$, we will obtain the following sum identities on Horadam numbers and its special cases.

Theorem 3.15.

$$
\sum_{k=0}^{n-1} W_{s+k t}= \begin{cases}\frac{W_{s}-W_{s+n t}-q^{s} U_{t-s}+q^{t} W_{s+(n-1) t}}{1-V_{t}+q^{t}}, & s<t \\ \frac{W_{s}-W_{(n+1) s}-q^{s}\left[a-W_{n s}\right]}{1-V_{s}+q^{s}}, & s=t \\ \frac{W_{s}-W_{s+n t}-q^{t}\left[W_{s-t}-W_{s+(n-1) t}\right]}{1-V_{t}+q^{t}}, & s>t\end{cases}
$$




\section{Corollary 3.16.}

$$
\sum_{k=0}^{n-1} F_{s+k t}= \begin{cases}\frac{F_{s}-F_{s+n t}+(-1)^{s+1} F_{t-s}+(-1)^{t} F_{s+(n-1) t}}{1-L_{t}+(-1)^{t}}, & s<t \\ \frac{F_{s}-F_{(n+1) s}+(-1)^{s} F_{n s}}{1-L_{s}+(-1)^{s}}, & s=t \\ \frac{F_{s}-F_{s+n t}-(-1)^{t}\left[F_{s-t}-F_{s+(n-1) t}\right]}{1-L_{t}+(-1)^{t}}, & s>t\end{cases}
$$

\section{Corollary 3.17.}

$$
\sum_{k=0}^{n-1} L_{s+k t}= \begin{cases}\frac{L_{s}-L_{s+n t}+(-1)^{s+1} L_{t-s}+(-1)^{t} L_{s+(n-1) t}}{1-L_{t}+(-1)^{t}}, & s<t \\ \frac{L_{s}-L_{(n+1) s}-(-1)^{s}\left[2-L_{n s}\right]}{1-L_{s}+(-1)^{s}}, & s=t \\ \frac{L_{s}-L_{s+n t}-(-1)^{t}\left[L_{s-t}-L_{s+(n-1) t}\right]}{1-L_{t}+(-1)^{t}}, & s>t\end{cases}
$$

\section{Corollary 3.18.}

$$
\sum_{k=0}^{n-1} J_{s+k t}= \begin{cases}\frac{J_{s}-J_{s+n t}+(-2)^{s} J_{t-s}+(-2)^{t} J_{s+(n-1) t}}{1-K_{t}+(-2)^{t}}, & s<t \\ \frac{J_{s}-J_{(n+1) s}+(-2)^{s} J_{n s}}{1-K_{s}+(-2)^{s}}, & s=t \\ \frac{J_{s}-J_{s+n t}-(-2)^{t}\left[J_{s-t}-J_{s+(n-1) t}\right]}{1-K_{t}+(-2)^{t}}, & s>t\end{cases}
$$

Corollary 3.19.

$$
\sum_{k=0}^{n-1} K_{s+k t}= \begin{cases}\frac{K_{s}-K_{s+n t}+(-2)^{s} K_{t-s}+(-2)^{t} K_{s+(n-1) t}}{1-K_{t}+(-2)^{t}}, & s<t \\ \frac{K_{s}-K_{(n+1) s}-(-2)^{s}\left[2-K_{n s}\right]}{1-K_{s}+(-2)^{s}}, & s=t \\ \frac{K_{s}-K_{s+n t}-(-2)^{t}\left[K_{s-t}-K_{s+(n-1) t}\right]}{1-K_{t}+(-2)^{t}}, & s>t\end{cases}
$$

\section{Corollary 3.20.}

$$
\sum_{k=0}^{n-1} P_{s+k t}= \begin{cases}\frac{P_{s}-P_{s+n t}+(-1)^{s} U_{t-s}+(-1)^{t} P_{s+(n-1) t}}{1-Q_{t}+(-1)^{t}}, & s<t \\ \frac{P_{s}-P_{(n+1) s}+(-1)^{s} P_{n s}}{1-Q_{s}+(-1)^{s}}, & s=t \\ \frac{P_{s}-P_{s+n t}-(-1)^{t}\left[P_{s-t}-P_{s+(n-1) t}\right]}{1-Q_{t}+(-1)^{t}}, & s>t\end{cases}
$$




\section{Corollary 3.21.}

$$
\sum_{k=0}^{n-1} Q_{s+k t}= \begin{cases}\frac{Q_{s}-Q_{s+n t}+(-1)^{s+1} Q_{t-s}+(-1)^{t} Q_{s+(n-1) t}}{1-Q_{t}+(-1)^{t}}, & s<t \\ \frac{Q_{s}-Q_{(n+1) s}-(-1)^{s}\left[2-Q_{n s}\right]}{1-Q_{s}+(-1)^{s}}, & s=t \\ \frac{Q_{s}-Q_{s+n t}-(-1)^{t}\left[Q_{s-t}-Q_{s+(n-1) t}\right]}{1-Q_{t}+(-1)^{t}}, & s>t\end{cases}
$$

\subsection{Results on divisibility}

From the results on determinant and sum identities, we now derive results on divisibility.

Theorem 3.22. Let $a, b, p, q, r \in \mathbb{Z}$.

1. If $s<t$, then $1-r V_{n t}+r^{2} q^{n t}$ divides $\left(W_{s}-r W_{s+n t}\right)^{n}-r\left[q^{s} U_{t-s}-r q^{t} W_{s+(n-1) t}\right]^{n}$.

2. If $s=t$, then $1-r V_{n s}+r^{2} q^{n s}$ divides $\left(W_{s}-r W_{(n+1) s}\right)^{n}-r q^{n s}\left[a-r W_{n s}\right]^{n}$.

3. If $s>t$, then $1-r V_{n t}+r^{2} q^{n t}$ divides $\left(W_{s}-r W_{s+n t}\right)^{n}-r q^{n t}\left[W_{s-t}-r W_{s+(n-1) t}\right]^{n}$.

Proof. Let $a, b, p, q, r \in \mathbb{Z}$. This means that $M(r, \vec{W})$ is an integer matrix. Hence its determinant $\Delta[r, \vec{W}]$ is an integer because integer matrices have integer determinants. From this, Theorem 3.22 immediately follows.

Here are the special cases of Theorem 3.22.

Corollary 3.23. Let $r \in \mathbb{Z}$.

1. If $s<t$, then $1-r L_{n t}+(-1)^{n t} r^{2}$ divides $\left(F_{s}-r F_{s+n t}\right)^{n}-r\left[(-1)^{s} F_{t-s}-(-1)^{t} r F_{s+(n-1) t}\right]^{n}$.

2. If $s=t$, then $1-r L_{n s}+(-1)^{n s} r^{2}$ divides $\left(F_{s}-r F_{(n+1) s}\right)^{n}-(-1)^{n s} r\left(-r F_{n s}\right)^{n}$.

3. If $s>t$, then $1-r L_{n t}+(-1)^{n t} r^{2}$ divides $\left(F_{s}-r F_{s+n t}\right)^{n}-(-1)^{n t} r\left(F_{s-t}-r F_{s+(n-1) t}\right)^{n}$.

Corollary 3.24. Let $r \in \mathbb{Z}$.

1. If $s<t$, then $1-r L_{n t}+(-1)^{n t} r^{2}$ divides $\left(L_{s}-r L_{s+n t}\right)^{n}-r\left[(-1)^{s} L_{t-s}-(-1)^{t} r L_{s+(n-1) t}\right]^{n}$.

2. If $s=t$, then $1-r L_{n s}+(-1)^{n s} r^{2}$ divides $\left(L_{s}-r L_{(n+1) s}\right)^{n}-(-1)^{n s} r\left[2-r L_{n s}\right]^{n}$.

3. If $s>t$, then $1-r L_{n t}+(-1)^{n t} r^{2}$ divides $\left(L_{s}-r L_{s+n t}\right)^{n}-(-1)^{n t} r\left[L_{s-t}-r L_{s+(n-1) t}\right]^{n}$.

Corollary 3.25. Let $r \in \mathbb{Z}$.

1. If $s<t$, then $1-r K_{n t}+(-2)^{n t} r^{2}$ divides $\left(J_{s}-r J_{s+n t}\right)^{n}-r\left[(-2)^{s+1} J_{t-s}-(-2)^{t} r J_{s+(n-1) t}\right]^{n}$.

2. If $s=t$, then $1-r K_{n s}+(-2)^{n s} r^{2}$ divides $\left(J_{s}-r J_{(n+1) s}\right)^{n}-(-2)^{n s} r\left(-r J_{n s}\right)^{n}$.

3. If $s>t$, then $1-r K_{n t}+(-2)^{n t} r^{2}$ divides $\left(J_{s}-r J_{s+n t}\right)^{n}-(-2)^{n t} r\left[J_{s-t}-r J_{s+(n-1) t}\right]^{n}$. 
Corollary 3.26. Let $r \in \mathbb{Z}$.

1. If $s<t$, then $1-r K_{n t}+(-2)^{n t} r^{2}$ divides $\left(K_{s}-r K_{s+n t}\right)^{n}-r\left[(-2)^{s} K_{t-s}-(-2)^{t} r K_{s+(n-1) t}\right]^{n}$.

2. If $s=t$, then $1-r K_{n s}+(-2)^{n s} r^{2}$ divides $\left(K_{s}-r K_{(n+1) s}\right)^{n}-(-2)^{n s} r\left[2-r K_{n s}\right]^{n}$.

3. If $s>t$, then $1-r K_{n t}+(-2)^{n t} r^{2}$ divides $\left(K_{s}-r K_{s+n t}\right)^{n}-(-2)^{n t} r\left[K_{s-t}-r K_{s+(n-1) t}\right]^{n}$.

Corollary 3.27. Let $r \in \mathbb{Z}$.

1. If $s<t$, then $1-r Q_{n t}+(-1)^{n t} r^{2}$ divides $\left(P_{s}-r P_{s+n t}\right)^{n}-r\left[(-1)^{s+1} P_{t-s}-(-1)^{t} r P_{s+(n-1) t}\right]^{n}$.

2. If $s=t$, then $1-r Q_{n s}+(-1)^{n s} r^{2}$ divides $\left(P_{s}-r P_{(n+1) s}\right)^{n}-(-1)^{n s} r\left(-r P_{n s}\right)^{n}$.

3. If $s>t$, then $1-r Q_{n t}+(-1)^{n t} r^{2}$ divides $\left(P_{s}-r P_{s+n t}\right)^{n}-(-1)^{n t} r\left[P_{s-t}-r P_{s+(n-1) t}\right]^{n}$.

Corollary 3.28. Let $r \in \mathbb{Z}$.

1. If $s<t$, then $1-r Q_{n t}+(-1)^{n t} r^{2}$ divides $\left(Q_{s}-r Q_{s+n t}\right)^{n}-r\left[(-1)^{s} Q_{t-s}-(-1)^{t} r Q_{s+(n-1) t}\right]^{n}$.

2. If $s=t$, then $1-r Q_{n s}+(-1)^{n s} r^{2}$ divides $\left(Q_{s}-r Q_{(n+1) s}\right)^{n}-(-1)^{n s} r\left[2-r Q_{n s}\right]^{n}$.

3. If $s>t$, then $1-r Q_{n t}+(-1)^{n t} r^{2}$ divides $\left(Q_{s}-r Q_{s+n t}\right)^{n}-(-1)^{n t} r\left[Q_{s-t}-r Q_{s+(n-1) t}\right]^{n}$.

Theorem 3.29. Let $a, b, p, q \in \mathbb{Z}$ and $r=1$.

1. If $s<t$, then $1-V_{t}+q^{t}$ divides $W_{s}-W_{s+n t}-q^{s} U_{t-s}+q^{t} W_{s+(n-1) t}$.

2. If $s=t$, then $1-V_{s}+q^{s}$ divides $W_{s}-W_{(n+1) s}-q^{s}\left[a-W_{n s}\right]$.

3. If $s>t$, then $1-V_{t}+q^{t}$ divides $W_{s}-W_{s+n t}-q^{t}\left[W_{s-t}-W_{s+(n-1) t}\right]$.

Proof. If $a, b, p, q \in \mathbb{Z}$ and $r=1$, we obtain the sum identity from Theorem 3.15 where the $W_{s+k t}$-s are all integers. Hence the sum is an integer, so the theorem immediately follows.

For the special cases, we have the following.

Corollary 3.30. Let $r=1$.

1. If $s<t$, then $1-L_{t}+(-1)^{t}$ divides $F_{s}-F_{s+n t}+(-1)^{s+1} F_{t-s}+(-1)^{t} F_{s+(n-1) t}$.

2. If $s=t$, then $1-L_{s}+(-1)^{s}$ divides $F_{s}-F_{(n+1) s}+(-1)^{s} F_{n s}$.

3. If $s>t$, then $1-L_{t}+(-1)^{t}$ divides $F_{s}-F_{s+n t}-(-1)^{t}\left[F_{s-t}-F_{s+(n-1) t}\right]$.

Corollary 3.31. Let $r=1$.

1. If $s<t$, then $1-L_{t}+(-1)^{t}$ divides $L_{s}-L_{s+n t}+(-1)^{s+1} L_{t-s}+(-1)^{t} L_{s+(n-1) t}$.

2. If $s=t$, then $1-L_{s}+(-1)^{s}$ divides $L_{s}-L_{(n+1) s}-(-1)^{s}\left[2-L_{n s}\right]$.

3. If $s>t$, then $1-L_{t}+(-1)^{t}$ divides $L_{s}-L_{s+n t}-(-1)^{t}\left[L_{s-t}-L_{s+(n-1) t}\right]$. 
Corollary 3.32. Let $r=1$.

1. If $s<t$, then $1-K_{t}+(-2)^{t}$ divides $J_{s}-J_{s+n t}+(-2)^{s} J_{t-s}+(-2)^{t} J_{s+(n-1) t}$.

2. If $s=t$, then $1-K_{s}+(-2)^{s}$ divides $J_{s}-J_{(n+1) s}+(-2)^{s} J_{n s}$.

3. If $s>t$, then $1-K_{t}+(-2)^{t}$ divides $J_{s}-J_{s+n t}-(-2)^{t}\left[J_{s-t}-J_{s+(n-1) t}\right]$.

Corollary 3.33. Let $r=1$.

1. If $s<t$, then $1-K_{t}+(-2)^{t}$ divides $K_{s}-K_{s+n t}+(-2)^{s} K_{t-s}+(-2)^{t} K_{s+(n-1) t}$.

2. If $s=t$, then $1-K_{s}+(-2)^{s}$ divides $K_{s}-K_{(n+1) s}-(-2)^{s}\left[2-K_{n s}\right]$.

3. If $s>t$, then $1-K_{t}+(-2)^{t}$ divides $K_{s}-K_{s+n t}-(-2)^{t}\left[K_{s-t}-K_{s+(n-1) t}\right]$.

Corollary 3.34. Let $r=1$.

1. If $s<t$, then $1-Q_{t}+(-1)^{t}$ divides $P_{s}-P_{s+n t}+(-1)^{s} U_{t-s}+(-1)^{t} P_{s+(n-1) t}$.

2. If $s=t$, then $1-Q_{s}+(-1)^{s}$ divides $P_{s}-P_{(n+1) s}+(-1)^{s} P_{n s}$.

3. If $s>t$, then $1-Q_{t}+(-1)^{t}$ divides $P_{s}-P_{s+n t}-(-1)^{t}\left[P_{s-t}-P_{s+(n-1) t}\right]$.

Corollary 3.35. Let $r=1$.

1. If $s<t$, then $1-Q_{t}+(-1)^{t}$ divides $Q_{s}-Q_{s+n t}+(-1)^{s+1} Q_{t-s}+(-1)^{t} Q_{s+(n-1) t}$.

2. If $s=t$, then $1-Q_{s}+(-1)^{s}$ divides $Q_{s}-Q_{(n+1) s}-(-1)^{s}\left[2-Q_{n s}\right]$.

3. If $s>t$, then $1-Q_{t}+(-1)^{t}$ divides $Q_{s}-Q_{s+n t}-(-1)^{t}\left[Q_{s-t}-Q_{s+(n-1) t}\right]$.

\subsection{Norms of $M(r, \vec{W})$}

For the Euclidean norm, we have the following results.

Theorem 3.36.

$$
\begin{aligned}
\|M(r, \vec{W})\|_{E}^{2}= & (A+B)\left[n \Omega_{1}-\left(1-|r|^{2}\right) \Omega_{2}\right]-A B\left[n \Omega_{3}-\left(1-|r|^{2}\right) \Omega_{4}\right] \\
& -2 A B\left[n \Omega_{5}-\left(1-|r|^{2}\right) \Omega_{6}\right]
\end{aligned}
$$

where

$$
\text { - } \Omega_{1}= \begin{cases}\frac{W_{2 s}-W_{2(s+n t)}-q^{2 s} U_{2(t-s)}+q^{2 t} W_{2(s+(n-1)) t}}{1-V_{2 t}+q^{2 t}}, & s<t \\ \frac{W_{2 s}-W_{2(n+1) s}-q^{2 s}\left[a-W_{2 n s}\right]}{1-V_{2 s}+q^{2 s}}, & s=t \\ \frac{W_{2 s}-W_{2(s+n t)}-q^{2 t}\left[W_{2(s-t)}-W_{2(s+(n-1)) t}\right]}{1-V_{2 t}+q^{2 t}}, & s>t\end{cases}
$$


- $\Omega_{2}=\frac{\gamma_{1}(s, t)-\gamma_{2}(s, t)}{\left(1-V_{2 t}+q^{2 t}\right)^{2}}$

- $\Omega_{3}= \begin{cases}\frac{V_{2 s}-V_{2(s+n t)}-q^{2 s} V_{2(t-s)}+q^{2 t} V_{2(s+(n-1)) t}}{1-V_{2 t}+q^{2 t}}, & s<t \\ \frac{V_{2 s}-V_{2(n+1) s}-q^{2 s}\left[2-V_{2 n s}\right]}{1-V_{2 s}+q^{2 s}}, & s=t \\ \frac{V_{2 s}-V_{2(s+n t)}-q^{2 t}\left[V_{2(s-t)}-V_{2(s+(n-1)) t}\right]}{1-V_{2 t}+q^{2 t}}, & s>t\end{cases}$

- $\Omega_{4}=\frac{\sigma_{1}(s, t)-\sigma_{2}(s, t)}{\left(1-V_{2 t}+q^{2 t}\right)^{2}}$

- $\Omega_{5}=\frac{q^{s}-q^{s+n t}}{1-q^{t}}$

- $\Omega_{6}=\frac{q^{s+t}-n q^{s+n t}+(n-1) q^{s+(n+1) t}}{\left(1-q^{t}\right)^{2}}$

- $\gamma_{1}(s, t)= \begin{cases}\left(W_{2 s}-W_{2(s+n t)}-q^{2 s} U_{2(t-s)}+q^{2 t} W_{2(s+(n-1) t}\right)\left(V_{2 t}-2 q^{2 t}\right), & s<t \\ \left(W_{2 s}-W_{2(n+1) s}-q^{2 s}\left(a-W_{2 n s}\right)\right)\left(V_{2 s}-2 q^{2 s}\right), & s=t \\ \left(W_{2 s}-W_{2(s+n t)}-q^{2 t}\left(W_{2(s-t)}-W_{2(s+(n-1) t)}\right)\right)\left(V_{2 t}-2 q^{2 t}\right), & s>t\end{cases}$

- $\gamma_{2}(s, t)= \begin{cases}\left(q^{2 s} U_{2(t-s)}+n W_{2(s+n t)}-(n+1) q^{2 t} W_{2(s+(n-1) t}\right)\left(1-V_{2 t}+q^{2 t}\right), & s<t \\ \left(a q^{2 s}+n W_{2(n+1) s}-(n+1) q^{2 s} W_{n s}\right)\left(1-V_{2 s}+q^{2 s}\right), & s=t \\ \left(q^{2 t} W_{2(s-t)}+n W_{2(s+n t)}-(n+1) q^{2 t} W_{2(s+(n-1) t)}\right)\left(1-V_{2 t}+q^{2 t}\right), & s>t\end{cases}$

- $\sigma_{1}(s, t)= \begin{cases}\left(V_{2 s}-V_{2(s+n t)}-q^{2 s} V_{2(t-s)}+q^{2 t} V_{2(s+(n-1) t)}\right)\left(V_{2 t}-2 q^{2 t}\right), & s<t \\ \left(V_{2 s}-V_{2(n+1) s}-q^{2 s}\left(2-V_{2 n s}\right)\right)\left(V_{2 s}-2 q^{2 s}\right), & s=t \\ \left(V_{2 s}-V_{2(s+n t)}-q^{2 t}\left(V_{2(s-t)}-V_{2(s+(n-1) t)}\right)\right)\left(V_{2 t}-2 q^{2 t}\right), & s>t\end{cases}$

- $\sigma_{2}(s, t)= \begin{cases}\left(q^{2 s} V_{2(t-s)}+n V_{2(s+n t)}-(n+1) q^{2 t} V_{2(s+(n-1) t)}\right)\left(1-V_{2 t}+q^{2 t}\right), & s<t \\ \left(2 q^{2 s}+n V_{2(n+1) s}-(n+1) q^{2 s} V_{2 n s}\right)\left(1-V_{2 s}+q^{2 s}\right), & s=t \\ \left(q^{2 t} V_{2(s-t)}+n V_{2(s+n t)}-(n+1) q^{2 t} V_{2(s+(n-1) t)}\right)\left(1-V_{2 t}+q^{2 t}\right), & s>t\end{cases}$ 
Proof.

$$
\begin{aligned}
& \sum_{k=0}^{n-1}\left|c_{k}\right|^{2}\left[n-k\left(1-|r|^{2}\right)\right]=\sum_{k=0}^{n-1} W_{s+k t}^{2}\left[n-k\left(1-|r|^{2}\right)\right] \\
& =\sum_{k=0}^{n-1}\left[A \alpha^{s+k t}+B \beta^{s+k t}\right]^{2}\left[n-k\left(1-|r|^{2}\right)\right] \\
& =\sum_{k=0}^{n-1}\left[A^{2} \alpha^{2(s+k t)}+2 A B q^{s+k t}+B^{2} \beta^{2(s+k t)}\right]\left[n-k\left(1-|r|^{2}\right)\right] \\
& =\sum_{k=0}^{n-1}\left[A^{2} \alpha^{2(s+k t)}+\left(A B \beta^{2(s+k t)}-A B \beta^{2(s+k t)}\right)\right]\left[n-k\left(1-|r|^{2}\right)\right] \\
& +\sum_{k=0}^{n-1}\left[B^{2} \beta^{2(s+k t)}+\left(A B \alpha^{2(s+k t)}-A B \alpha^{2(s+k t)}\right)\right]\left[n-k\left(1-|r|^{2}\right)\right] \\
& +2 A B \sum_{k=0}^{n-1} q^{s+k t}\left[n-k\left(1-|r|^{2}\right)\right] \\
& =\sum_{k=0}^{n-1}\left[A\left(A \alpha^{2(s+k t)}+B \beta^{2(s+k t)}\right)\right]\left[n-k\left(1-|r|^{2}\right)\right] \\
& +\sum_{k=0}^{n-1}\left[B\left(A \alpha^{2(s+k t)}+B \beta^{2(s+k t)}\right)\right]\left[n-k\left(1-|r|^{2}\right)\right] \\
& -\sum_{k=0}^{n-1}\left[A B\left(\alpha^{2(s+k t)}+\beta^{2(s+k t)}\right)\right]\left[n-k\left(1-|r|^{2}\right)\right] \\
& +\sum_{k=0}^{n-1}\left[2 A B q^{s+k t}\right]\left[n-k\left(1-|r|^{2}\right)\right] \\
& =\sum_{k=0}^{n-1}\left[(A+B) W_{2(s+k t)}-A B V_{2(s+k t)}+2 A B q^{s+k t}\right]\left[n-k\left(1-|r|^{2}\right)\right] \\
& =n \sum_{k=0}^{n-1}\left[(A+B) W_{2(s+k t)}-A B V_{2(s+k t)}+2 A B q^{s+k t}\right] \\
& -\left(1-\left|r^{2}\right|\right) \sum_{k=0}^{n-1}\left[(A+B) k W_{2(s+k t)}-A B k V_{2(s+k t)}+2 A B k q^{s+k t}\right] .
\end{aligned}
$$

Using the sum identities that we have derived and the sum of finite geometric sequence, we obtain the following expression from the first summation:

$$
n \sum_{k=0}^{n-1}\left[(A+B) W_{2(s+k t)}-A B V_{2(s+k t)}+2 A B q^{s+k t}\right]=(A+B) n \Omega_{1}-A B n \Omega_{3}-2 A B n \Omega_{5}
$$


For the second summation, we use the following finite generating functions

$$
\begin{aligned}
& F_{s, t}(x)=\sum_{k=0}^{n-1} W_{s+k t} x^{k}= \begin{cases}\frac{W_{s}-q^{s} U_{t-s} x-W_{s+n t} x^{n}+q^{t} W_{s+(n-1) t} x^{n+1}}{1-V_{t} x+q^{t} x^{2}}, & s<t \\
\frac{W_{s}-a q^{s} x-W_{(n+1) s} x^{n}+q^{s} W_{n s} x^{n+1}}{1-V_{s} x+q^{s} x^{2}}, & s=t \\
\frac{W_{s}-q^{t} W_{s-t} x-W_{s+n t} x^{n}+q^{t} W_{s+(n-1) t} x^{n+1}}{1-V_{t} x+q^{t} x^{2}}, & s>t\end{cases} \\
& G_{s, t}(x)=\sum_{k=0}^{n-1} V_{s+k t} x^{k}= \begin{cases}\frac{V_{s}-q^{s} V_{t-s} x-V_{s+n t} x^{n}+q^{t} V_{s+(n-1) t} x^{n+1}}{1-V_{t} x+q^{t} x^{2}}, & s<t \\
\frac{V_{s}-2 q^{s} x-V_{(n+1) s} x^{n}+q^{s} V_{n s} x^{n+1}}{1-V_{s} x+q^{s} x^{2}}, & s=t \\
\frac{V_{s}-q^{t} V_{s-t} x-V_{s+n t} x^{n}+q^{t} V_{s+(n-1) t} x^{n+1}}{1-V_{t} x+q^{t} x^{2}}, & s>t\end{cases} \\
& H_{s, t}(x)=\sum_{k=0}^{n-1} q^{s+k t} x^{k}=\frac{q^{s}-q^{s+n t} x^{n} .}{1-q^{t} x} .
\end{aligned}
$$

Consequently, we have

$$
\begin{aligned}
& \left(1-\left|r^{2}\right|\right) \sum_{k=0}^{n-1}\left[(A+B) k W_{2(s+k t)}-A B k V_{2(s+k t)}+2 A B k q^{s+k t}\right] \\
= & \left(1-\left|r^{2}\right|\right)\left[(A+B) F_{2 s, 2 t}^{\prime}(1)-A B G_{2 s, 2 t}^{\prime}(1)+2 A B H_{s, t}^{\prime}(1)\right] \\
= & (A+B)\left(1-|r|^{2}\right) \Omega_{2}-A B\left(1-|r|^{2}\right) \Omega_{4}+2 A B\left(1-|r|^{2}\right) \Omega_{6} .
\end{aligned}
$$

Hence we have

$$
\begin{aligned}
\|M(r, \vec{W})\|_{E}^{2}= & (A+B)\left[n \Omega_{1}-\left(1-|r|^{2}\right) \Omega_{2}\right]-A B\left[n \Omega_{3}-\left(1-|r|^{2}\right) \Omega_{4}\right] \\
& -2 A B\left[n \Omega_{5}-\left(1-|r|^{2}\right) \Omega_{6}\right] .
\end{aligned}
$$

For $r= \pm 1$ we have the following result.

\section{Corollary 3.37.}

$$
\|M(1, \vec{W})\|_{E}^{2}=n\left[(A+B) \Omega_{1}-A B \Omega_{3}-2 A B \Omega_{5}\right]=\|M(-1, \vec{W})\|_{E}^{2} .
$$

For the spectral norm, we have the following results.

\section{Theorem 3.38.}

$$
\begin{aligned}
& \|M(r, \vec{W})\|_{2} \\
& =\max _{0 \leq m \leq n-1} \sqrt{\frac{\left[\delta_{1}(r, s, t)\right]^{2}-2 \delta_{1}(r, s, t) \delta_{2}(r, s, t)|r|^{1 / n} \cos \tau_{m}+\left[\delta_{2}(r, s, t)\right]^{2}|r|^{2 / n}}{1-2 V_{t}|r|^{1 / n} \cos \tau_{m}\left(1+q^{t}|r|^{2 / n}\right)+|r|^{2 / n}\left(4 q^{t} \cos ^{2} \tau_{m}+V_{2 t}+q^{2 t}|r|^{2 / n}\right)}}
\end{aligned}
$$

where: 
- $\tau_{m}=\frac{\theta+2(j-m) \pi}{n}$,

- $\delta_{1}(r, s, t)=\left\{\begin{array}{ll}W_{s}-r W_{s+n t}, & s \neq t \\ W_{s}-r W_{(n+1) s}, & s=t\end{array}\right.$,

- $\delta_{2}(r, s, t)= \begin{cases}q^{s} U_{t-s}-r q^{t} W_{s+(n-1) t}, & s<t \\ q^{s}\left[a-r W_{n s}\right], & s=t \\ q^{t}\left[W_{s-t}-r W_{s+(n-1) t}\right], & s>t\end{cases}$

Proof. Let $\tau_{m}=\frac{\theta+2(j-m) \pi}{n}, \delta_{1}(r, s, t)=\left\{\begin{array}{ll}W_{s}-r W_{s+n t}, & s \neq t \\ W_{s}-r W_{(n+1) s}, & s=t\end{array}\right.$ and $\delta_{2}(r, s, t)= \begin{cases}q^{s} U_{t-s}-r q^{t} W_{s+(n-1) t}, & s<t \\ q^{s}\left[a-r W_{n s}\right], & s=t . \\ q^{t}\left[W_{s-t}-r W_{s+(n-1) t}\right], & s>t\end{cases}$

Computing the spectral norm, we have

$$
\|M(r, \vec{W})\|_{2}=\max _{0 \leq m \leq n-1}\left|\frac{\delta_{1}(r, s, t)-\delta_{2}(r, s, t) \rho \omega^{-m}}{\left(1-\alpha^{t} \rho \omega^{-m}\right)\left(1-\beta^{t} \rho \omega^{-m}\right)}\right| .
$$

Note that for any $M, N \in \mathbb{R}$, we have the following equation.

$$
\begin{aligned}
\left|M-N \rho \omega^{-m}\right| & =\left.|M-N| r\right|^{1 / n}\left[\cos \left(\frac{\theta+2 \pi(j-m)}{n}\right)+i \sin \left(\frac{\theta+2 \pi(j-m)}{n}\right)\right] \mid \\
& =\left.|M-N| r\right|^{1 / n}\left[\cos \tau_{m}+i \sin \tau_{m}\right] \mid \\
& =\sqrt{M^{2}-2 M N|r|^{1 / n} \cos \tau_{m}+N^{2}|r|^{2 / n}}
\end{aligned}
$$

Applying the norm equation above, results to:

$$
\begin{aligned}
& \|M(r, \vec{W})\|_{2} \\
& =\max _{0 \leq m \leq n-1} \sqrt{\frac{\left[\delta_{1}(r, s, t)\right]^{2}-2 \delta_{1}(r, s, t) \delta_{2}(r, s, t)|r|^{1 / n} \cos \tau_{m}+\left[\delta_{2}(r, s, t)\right]^{2}|r|^{2 / n}}{1-2 V_{t}|r|^{1 / n} \cos \tau_{m}\left(1+q^{t}|r|^{2 / n}\right)+|r|^{2 / n}\left(4 q^{t} \cos ^{2} \tau_{m}+V_{2 t}+q^{2 t}|r|^{2 / n}\right)}} .
\end{aligned}
$$

For the cases $r= \pm 1$, we have the following results.

\section{Corollary 3.39.}

$$
\|M(1, \vec{W})\|_{2}=\max _{0 \leq m \leq n-1} \sqrt{\frac{\left[\delta_{1}(1, s, t)\right]^{2}-2 \delta_{1}(1, s, t) \delta_{2}(1, s, t) \cos \tau_{m}+\left[\delta_{2}(1, s, t)\right]^{2}}{1-2 V_{t} \cos \tau_{m}\left(1+q^{t}\right)+4 q^{t} \cos ^{2} \tau_{m}+V_{2 t}+q^{2 t}}} .
$$

Corollary 3.40.

$$
\|M(-1, \vec{W})\|_{2}=\max _{0 \leq m \leq n-1} \sqrt{\frac{\left[\delta_{1}(-1, s, t)\right]^{2}-2 \delta_{1}(-1, s, t) \delta_{2}(-1, s, t) \cos \tau_{m}+\left[\delta_{2}(-1, s, t)\right]^{2}}{1-2 V_{t} \cos \tau_{m}\left(1+q^{t}\right)+4 q^{t} \cos ^{2} \tau_{m}+V_{2 t}+q^{2 t}}} .
$$




\section{Conclusion and recommendations}

We have obtained the explicit forms of the eigenvalues, determinants, Euclidean norms and spectral norms of $r$-circulant matrices whose entries are Horadam numbers having arithmetic indices. Special cases (Fibonacci, Lucas, Jaconsthal, Jacobsthal-Lucas, Pell and Pell-Lucas) were also obtained for the eigenvalues and determinants. Special cases where $r= \pm 1$ were also obtained for the Euclidean norm and spectral norm. Furthermore, sum identities and divisibility results were also obtained. Lastly, we have generalized the results that involve eigenvalues, determinants, Euclidean norms and spectral norm in Bozkurt [2-4], Bueno [7-14], Civciv and Turkmen [16], and Lind [19].

For future work, we recommend the following:

- determine the inverse of $r$-circulant matrices with Horadam numbers having arithmetic indices;

- determine the eigenvalues, determinants, Euclidean norms and spectral norms of the matrix given by

$$
M[r, \vec{G}]=\left(\begin{array}{cccccc}
G_{s} & G_{s+t} & G_{s+2 t} & \cdots & G_{s+(n-2) t} & G_{s+(n-1) t} \\
r G_{s+(n-1) t} & G_{s} & G_{s+t} & \cdots & G_{s+(n-3) t} & G_{s+(n-2) t} \\
r G_{s+(n-2) t} & r G_{s+(n-1) t} & G_{s} & \cdots & G_{s+(n-4) t} & G_{s+(n-3) t} \\
\vdots & \vdots & \vdots & \ddots & \vdots & \vdots \\
r G_{s+2 t} & r G_{s+3 t} & r G_{s+4 t} & \cdots & G_{s} & G_{s+t} \\
r G_{s+t} & r G_{s+2 t} & r G_{s+3 t} & \cdots & r G_{s+(n-1) t} & G_{s}
\end{array}\right),
$$

where $G_{j}$-s are Generalized Fibonacci numbers.

\section{References}

[1] Bahsi, M., \& Solak, S. (2010). On the Circulant Matrices with Arithmetic Sequence, International Journal of Contemporary Mathematical Sciences, 5 (25), 1213-1222.

[2] Bozkurt, D. (2012). On the Determinants and Inverses of Circulant Matrices with a General Number Sequence, Preprint. arXiv: 1202.1068v1 [math.NA] 6 Feb 2012.

[3] Bozkurt, D., \& Tam, T. Y. (2012). Determinants and inverses of circulant matrices with Jacobsthal and Jacobsthal-Lucas numbers. Appl. Math. Comput., 219, 544-551.

[4] Bozkurt, D. (2012). On the Determinants and Inverses of Circulant Matrices with Pell and Pell-Lucas Numbers, Preprint. arXiv: 1201.6061v1 [math.NA] 29 Jan 2012.

[5] Bozkurt, D., \& Tam, T. Y. (2014). Determinants and inverses of $r$-circulant matrices associated with a number sequence, Linear and Multilinear Algebra, 63 (10), 2079-2088.

[6] Bueno, A. C. F. (2012). On Right Circulant Matrices with Geometric Progression, International Journal of Applied Mathematical Research, 1 (4), 593-603. 
[7] Bueno, A. C. F. (2012). Right Circulant Matrices with Fibonacci Sequence, International Journal of Mathematics and Scientific Computing, 2 (2), 9-10.

[8] Bueno, A. C. F. (2013). Right Circulant Matrices with Jacobsthal Sequence, International Journal of Advanced Mathematical Sciences, 1 (2), 87-90.

[9] Bueno, A. C. F. (2013). Generalized Right Circulant Matrices with Geometric Sequence, International Journal of Mathematics and Scientific Computing, 3 (1), 17-18.

[10] Bueno, A. C. F. (2014). On the Eigenvalues and the Determinant of the Right Circulant Matrices with Pell and Pell-Lucas Numbers, International Journal of Mathematics and Scientific Computing, 4 (1), 19-20.

[11] Bueno, A. C. F. (2014). Right Circulant Matrices with Sum of the Terms of Two Geometric Sequences, International Journal of Mathematics and Scientific Computing, 4 (2), 51-52.

[12] Bueno, A. C. F. (2016). Right circulant matrices with ratio of the elements of Fibonacci and geometric sequence, Notes on Number Theory and Discrete Mathematics, 22 (3), 79-83.

[13] Bueno, A. C. F. (2016). Right circulant determinant sequences with Jacobsthal and Jacobsthal-Lucas Numbers, Notes on Number Theory and Discrete Mathematics, 22 (4), 56-61.

[14] Bueno, A. C. F. (2017). On $r$-circulant matrices with Fibonacci and Lucas numbers having arithmetic indices, AIP Conference Proceedings 1905, Article No. 030010.

[15] Cline, R. E., Plemmons, R. J., \& Worm, G. (1974). Generalized Inverses of Certain Toeplitz Matrices, Linear Algebra and Its Applications, 8, 25-33.

[16] Civciv, H., \& Turkmen, R. (2008). Notes on Norms of Circulant Matrices with Lucas Numbers, International Journal of Information and System Sciences, 4 (1), 142-147.

[17] Gray, R. (2006). Toeplitz and Circulant Matrices: A Review. Foundations and Trends in Communications and Information Theory, 2 (3), 155-239. Available online at: https: //ee.stanford.edu/ gray/toeplitz.pdf.

[18] Horadam, A. F. (1965). Basic Properties of a Certain Generalised Sequence of Numbers, Fibonacci Quarterly 3, 161-176.

[19] Lind, D. A. (1970). A Fibonacci Circulant, Fibonacci Quarterly, 8, 449-455.

[20] Majumdar, A. A. K. (2010). Wandering in the World of Smarandache Numbers, InProQuest, Ann Arbor. Available online at: http: / / fs .gallup.unm. edu/Ma jumdar.pdf.

[21] Nalli, A., \& Sen, M. (2010). On the Norms of Circulant Matrices with Generalized Fibonacci Numbers, Selcuk Journal of Applied Mathematics, 11 (1), 107-116. 
[22] Radiçić, B. (2019). On k-circulant matrices involving geometric sequence, Hacet. J. Math. Stat., 48 (3), 805-817.

[23] Shen, S., \& Cen, J. (2011). On the Norms of Circulant Matrices with the $(k, h)$-Fibonacci and $(k, h)$-Lucas Numbers, International Journal of Contemporary Mathematics and Sciences, 6 (19), 887-894.

[24] Shen, S., \& Cen, J. (2010). On the Spectral Norms of $r$-Circulant Matrices with the $k$-Fibonacci and $k$-Lucas Numbers, International Journal of Contemporary Mathematics and Sciences, 5 (12), 569-578.

[25] Yalciner, A. (2008). Spectral Norms of Some Special Circulant Matrices, International Journal of Contemporary Mathematics and Sciences, 3 (35), 1733-1738. 